\title{
BENEFICIOS TRIBUTARIOS DEL SECTOR AGRARIO Y DESARROLLO ECONÓMICO-SOCIAL PERUANO
}

\author{
TAX BENEFITS OF THE AGRICULTURAL SECTOR AND PERUVIAN SOCIAL- \\ ECONOMIC DEVELOPMENT
}

\section{RESUMEN}

Objetivo: Determinar la relación entre los beneficios tributarios del sector agrario y el desarrollo económico-social peruano. Método: El estudio se basó en el método analítico y sintético de las relaciones de las variables cuantitativas investigadas bajo un diseño no experimental, utilizando como técnicas el análisis documental y el modelo estadístico de la correlación canónica, considerando la información pública en un periodo transversal (2005-2019). Resultados: El indicador de Hotellings evidencia una relación significativa de ambos grupos de variables; sin embargo, ante un análisis disgregado, las correlaciones muestran resultados cuestionables en comparación con los resultados del estado del arte del tema investigado. Conclusiones: Se muestran relaciones directas y significativas entre el producto bruto interno con las exportaciones del sector, la formalización tributaria y los pagos del impuesto a la renta empresarial. No existe una relación directa significativa entre los gastos EsSalud y la formalización laboral del sector, de igual manera, entre los gastos de tributos internos y la formalización laboral, sustentada en la prueba estadística de la correlación canónica entre las variables planteadas en la presente investigación.

Palabras clave: Beneficios tributarios; sector agrario peruano; gasto tributario.
Segundo Eloy Tuesta Bardalez Universidad Nacional de Cajamarca Cajamarca, Perú

ORCID: https://orcid.org/0000-0003-1224-2093 Autor para correspondencia: stuesta1977@gmail.com

Héctor Villegas Chávez Universidad Nacional de Cajamarca Cajamarca, Perú ORCID: https://orcid.org/0000-0003-2050-1294 Correo electrónico: hvillegas@unc.edu.pe

OrLando Roberto Yupangu Linares Universidad Nacional de Cajamarca Cajamarca, Perú

ORCID: https://orcid.org/0000-0001-6679-1253 Correo electrónico: ryupanqui@unc.edu.pe

[Recibido: 03/09/2021 Aceptado: 03/12/2021 Publicado: 29/12/2021]

\begin{abstract}
Objective: Determine the relationship between the agrarian sector's tax benefits and the Peruvian economic and social development. Method: The study was based on the analytical and synthetic method of the quantitative variables' relationships investigated under a non-experimental design, using documentary analysis and the statistical model of canonical correlation as techniques, considering public information, in a cross-sectional period (2005-2019). Results: The Hotellings indicator evidences a significant relationship of both groups of variables; however, before a disaggregated analysis, the correlations show questionable results compared to the results of the state of the art of the investigated topic. Conclusions: There are direct and significant relationships between the gross domestic product, the sector's exports, tax formalization and corporate income tax payments. There is no significant direct relationship between EsSalud Expenditures and the sector's Labor Formalization, as well as between the Internal Tax Expenditures and Labor Formalization, which is supported by the statistical test of the canonical correlation between the variables proposed in this research.
\end{abstract}

Keywords: Tax benefits; Peruvian agricultural sector; tax expenditure. 


\section{INTRODUCCIÓN}

El sector agrario representa una importante actividad productiva y estratégica para la economía peruana que, en los últimos quince años, ha mostrado un indicador importante de crecimiento en las exportaciones, sobre todo, en el rubro agroexportador, al dirigir sus productos a mercados competitivos como el asiático, europeo y americano, aprovechando los diversos beneficios tributarios del sistema y las ventajas ofrecidas por los diversos tratados de libre comercio suscritos por el Estado peruano.

Las cifras económicas del sector agrario evidencian un crecimiento sostenido de las agroexportaciones peruanas al superar los montos exportados en $400 \%$, durante el periodo 2005-2019 (Banco Central de Reserva del Perú, 2021). Las cifras son representativas del crecimiento económico del sector agroexportador, el cual aprovecha las ventajas productivas del territorio peruano concentradas en la zona costera del país.

En Ecuador, según el estudio de Chuncho, Uriguen y Apolo (2021), desde antes de la aparición del petróleo, el sector agropecuario ha sido el mayor sustento del país, motivado por el desarrollo del área rural que ocupa cerca del $40 \%$ de la población; asimismo, ha buscado lograr el cambio de los productos exportados de materias primas a productos elaborados con mayor valor agregado, a pesar de las políticas implementadas por el Estado ecuatoriano, cuyos resultados no han sido evaluados de forma objetiva, siendo necesario considerar un análisis integral con un enfoque social-económico.

En Chile, los resultados de la reforma agraria presentaron secuelas alejadas de la voluntad de los agricultores, lo que ocasionó el fraccionamiento de las tierras agrícolas. Esto debido a que, según Thezá, Flores y Gac (2017), se promovió una agricultura globalmente competitiva materializada en el crecimiento del rubro exportador, pero que no ha sido robusto ni inclusivo pese al desarrollo rural.

En Argentina, según Toledo (2017), a pesar del enfoque neoliberal de varios Gobiernos de turno, la política agraria no logró promover el sector rural, por lo que los efectos negativos de la problemática de los años noventa se evidencian en la situación actual de la economía del país.

En Alicante (España), Silva, Gómez y Nivaldo (2016) demostraron que las políticas públicas promovieron el desarrollo rural-agrario, así como el desarrollo de innovación tecnológica, de inversión en estructuras modernas y articulación económica-ambiental, siendo relevante la organización agrícola en las cooperativas, lo que representó un medio idóneo de acceso al competitivo mercado europeo.
En cuanto a la realidad del sector agrario nacional, Castellares, Regalado y Huarancca (2018) concluyen que las normas tributarias del mencionado sector (beneficios tributarios-laborales) han promovido de manera directa el crecimiento del sector agroexportador de productos agrícolas tradicionales y agropecuarios considerados como no tradicionales (espárragos, frutas, entre otros), posicionando y diversificando la oferta exportadora peruana y generando empleos formales con base en el uso de tecnologías innovadoras por las empresas exportadoras, junto con interesantes incentivos tributarios que ofrece el sistema con las actuales normas tributarias-laborales del sistema. Todo ello concuerda con las diferentes cifras económicas publicadas por los entes estatales competentes, las cuales han servido de sustento para la celebración de nuevos tratados de libre comercio por parte del Estado peruano, que busca impulsar el crecimiento de las agroexportaciones nacionales.

En relación con la implementación de políticas diferenciadas, Benavente, Camargo, Sarmiento y Mena (2018) señalan que estas son necesarias para superar los problemas estructurales del sector agrícola, como la división minoritaria de tierras, el alto riesgo productivo, el uso inadecuado de los recursos naturales (deforestación), la deficiente asistencia técnica, el limitado desarrollo de la cadena comercializadora y la presencia de entidades estatales con servicios agropecuarios inclusivos para atender a pequeños productores que operan en la economía peruana. Lo mencionado representa un problema estructural del sector agrícola y agropecuario peruano sobre todo en la pequeña agricultura, por lo que es relevante implementar las políticas adecuadas de acuerdo con la realidad problemática de los diferentes sectores productivos.

El desarrollo agrario en el Perú aún es incipiente y no inclusivo para los pequeños productores, siendo necesario el cambio hacia políticas inclusivas y descentralizadas promovidas por ministerios gestores, según la realidad problemática objetiva del sector (Barrantes, Salinas y Yagüe, 2017). En ese sentido, la problemática del desarrollo limitado de la pequeña agricultura, representada por la informalidad del sector agrario, se simboliza en un problema estructural de la economía peruana y es descrita en las citas sucesivas de la presente investigación.

Según cifras publicadas por el Instituto Nacional de Estadística e Informática (2019), en el Perú operan pequeños y medianos productores agrícolas, quienes suman cerca de 2170000 productores (391000 desarrollan actividad agrícola, 47000 pecuaria y 1730000 agropecuaria), de los cuales un elevado porcentaje son informales (más del $80 \%$ ), y se concentran, en su mayoría, en la sierra peruana. Las cifras citadas evidencian resultados desfavorables de las normas de promoción del sector agrario y 
sus beneficios tributarios-laborales para este segmento de productores que forma parte de la red empresarial de la economía peruana.

Ante lo expuesto, se pretende responder la siguiente pregunta de investigación: ¿Los beneficios tributarios del sector agrario tienen relación directa con el desarrollo económico-social peruano (2005-2019)? Para la cual se plantea como hipótesis general: "Los beneficios tributarios del sector agrario tienen relación directa con el desarrollo económico-social peruano".

La presente investigación considera como principales normas de promoción del sector agrario las detalladas en la Tabla 1, las cuales son creadoras de los diferentes beneficios tributarios-laborales que buscan promover el desarrollo del sector, fortaleciendo la formalización tributaria-laboral de las unidades productoras que operan en la economía peruana, incluyendo los pequeños productores hasta empresas medianas y grandes concentradas en el segmento agroexportador.

\section{MATERIAL Y MÉTODOS}

La investigación se basó en un enfoque cuantitativo que consistió en el análisis descriptivo de la información pública de los entes competentes como el Banco Central de Reserva del Perú (BCRP) y la Superintendencia Nacional de Aduanas y de Administración Tributaria (SUNAT), análisis materializado en tablas descriptivas y de tipo estadístico, considerando para su estudio el método analítico y sintético. Se utilizó la técnica del análisis documental de la información económica del periodo 2005-2019, excluyendo del análisis las cifras del año 2020 por posibles efectos indirectos de la pandemia del COVID-19.

Se utilizó el método multivariado de la correlación canónica, el cual, según Badii, Castillo, Cortez, Wong y Villalpando (2007), es el método más generalizado del conjunto de técnicas estadísticas multivariante; además, se relaciona de forma directa con varios métodos de dependencia, cuyo objetivo es cuantificar la validez de la relación entre dos conjuntos de variables y emplear cualquier variable métrica sin que cumpla el estricto supuesto de normalidad. En el presente estudio, se consideraron diferentes variables tributarias de los beneficios del sector agrario (detalladas en la Tabla 4), a fin de determinar la relación de estos y el desarrollo económico-social peruano. Cabe resaltar que el análisis a través del programa SPSS permitió identificar qué variables representaban las de mayor peso.

En un inicio, se recopiló información cualitativa de las principales normas del sector agrario peruano a fin de analizar sus contenidos y vigencia (Ver Tabla 1). Luego de ello, se accedió a información pública del Banco Central de Reserva del Perú (BCRP), sobre el producto bruto interno (PBI) y los montos de las exportaciones de los sectores agrícola y agropecuario, representando las primeras variables cuantitativas de interés que fueron complementadas con información publicada por la Superintendencia Nacional de Aduanas y de Administración Tributaria (SUNAT).

\section{RESULTADOS}

La Tabla 2 muestra la información del desempeño de variables económicas vinculadas con el sector agrario que evidencia el crecimiento sostenido del PBI agrario y las exportaciones del sector agroexportador, lo cual, al cierre del año 2019, constituye una participación significativa del PBI (más del 10\%), donde se muestra un crecimiento del 435\% al comparar los años de 2005 a 2019. Cabe precisar que este importante rubro agroexportador ha sido promovido por los beneficios tributarios de las normas del sector agrario y por los más de 20 tratados de libre comercio (TLC) suscritos por el Gobierno peruano con países de destino de los continentes antes mencionados, los mismos

Tabla 1

Principales beneficios tributarios-sector agrario

\begin{tabular}{llc}
\hline \multicolumn{1}{c}{ Normas legales } & \multicolumn{1}{c}{ Beneficio } & Vigencia \\
\hline Decreto Supremo No 055-99-EF (artículos 33 y 34) & $\begin{array}{l}\text { Saldo a favor del exportador (SFMB)- Exportaciones de bienes } \\
\text { exoneradas del IGV }\end{array}$ & $16 / 04 / 1999$ \\
& $\begin{array}{l}\text { Tasas rebajadas: impuesto a la renta empresarial (15\%), EsSalud } \\
\text { (4\%), un régimen laboral especial para empresas formales, entre } \\
\text { Ley No } 27360\end{array}$ & $1 / 01 / 2000$ \\
& $\begin{array}{l}\text { otros beneficios. } \\
\text { Drawback del 3\% del valor FOB exportado }\end{array}$ & $31 / 12 / 2020$ \\
Decreto Legislativo No 1053 & $\begin{array}{l}\text { Incentivos para el sector agrario agroexportador y el nuevo régi- } \\
\text { men laboral agrario }\end{array}$ & $1 / 01 / 2021$ \\
Ley No 31110 & & $28 / 06 / 2008$ \\
\hline
\end{tabular}

Fuente: Elaboración propia sobre la base de información pública de normas legales: Ley No 31110 (2020), Ley № 27360 (2000), Decreto Supremo № 055 99-EF (1999) y Decreto Legislativo № 1053 (2008) 
Tabla 2

Cifras económicas del sector agrario peruano (en millones de soles)

\begin{tabular}{ccccc}
\hline Años & PBI-Perú & PBI-Agrario (PBIA) & Exportaciones Agrario (EXP) & (EXP/PBIA) \\
\hline 2005 & 273971 & 33308 & 4406 & $13 \%$ \\
2006 & 294598 & 36245 & 5865 & $16 \%$ \\
2007 & 319693 & 37189 & 6160 & $17 \%$ \\
2008 & 348870 & 38284 & 7562 & $20 \%$ \\
2009 & 352693 & 38839 & 7390 & $19 \%$ \\
2010 & 382081 & 40289 & 8937 & $22 \%$ \\
2011 & 406256 & 42015 & 12418 & $30 \%$ \\
2012 & 431199 & 44851 & 10958 & $24 \%$ \\
2013 & 456435 & 45805 & 11334 & $25 \%$ \\
2014 & 467308 & 46675 & 14326 & $31 \%$ \\
2015 & 482506 & 48240 & 16292 & $34 \%$ \\
2016 & 501581 & 49460 & 18790 & $38 \%$ \\
2017 & 514215 & 50802 & 19344 & $38 \%$ \\
2018 & 534665 & 54878 & 21799 & $40 \%$ \\
2019 & 546161 & 56711 & 23592 & $42 \%$ \\
\hline
\end{tabular}

Nota. Las cifras en dólares americanos (U\$) se convirtieron a un tipo de cambio promedio de venta por años en soles publicado por el Banco Central de Reserva del Perú.

Fuente: Elaboración propia sobre la base del Banco Central de Reserva del Perú (2021)

que ofrecen importantes beneficios arancelarios y de simplificación de los procedimientos aduaneros.

Asimismo, la Tabla 2 evidencia el peso porcentual creciente de las exportaciones en el PBI agrario, migrando de un $13 \%$ en el año 2005 a $42 \%$ al cierre del año 2019. Este hecho constituye una primera gran evidencia del despegue del sector agroexportador, siendo relevante identificar los efectos colaterales en el desarrollo social y económico del país, los cuales son medidos con indicadores precisos en otras tablas de análisis de la presente investigación.

Por otro lado, la información que se muestra en la Tabla 3 comprende los resultados en cifras de las normas de promoción del sector agrario, de forma específica, de los gastos tributarios que asume el sistema tributario peruano para promover el sector, representados por los montos dejados de recaudar (gastos tributarios), siendo los principales beneficios el impuesto a la renta empresarial (tasa rebajada del $15 \%$ en vez del $30 \%$ en promedio), así como la contribución a EsSalud (4\% en vez de 9\%) asumida por los empleadores (empresas como personas naturales o jurídicas) y los beneficios de devolución de impuestos que gozan las empresas acogidas a las normas del sector agrario (detalladas en la Tabla 1). Dichos beneficios han sido reformulados por las modificatorias correspondientes a partir del año 2021 por la "Ley del Régimen Labo- ral Agrario y de Incentivos para el sector agrario y riego, agroexportador y agroindustrial" (Ley $\mathrm{N}^{\circ} 31110,2020$ ), motivadas por significativos conflictos sociales de los trabajadores del sector agrario a fines del año 2020.

Es importante señalar que, en plena pandemia del COVID-19, el referido sector ha continuado operando en la economía y exportando su producción a diferentes mercados del mundo.

La Tabla 3 evidencia el crecimiento continuo de dichas variables económicas por efecto de los beneficios tributarios ofrecidos por el sistema tributario. Al analizar las cifras del periodo 2005-2019, se muestra el comportamiento de los beneficios vinculados con el impuesto general a las ventas (IGV) e impuesto a la renta empresarial (IRE), mientras que los beneficios de devolución del IGV han crecido 435\%, y el gasto del IRE se incrementó en un 160\%. Dichas cifras no guardan una correlación directa, dado que a mayor PBI y exportaciones del sector, se debería presentar un mayor pago del impuesto a la renta empresarial determinado y declarado por las empresas acogidas del sector agrario.

Al cierre del año 2019, 2417 empresas se acogieron a los beneficios del sector agrario, donde 997 registraron actividades de comercio exterior (agroexportadores) y de las cuales 337 empresas son consideradas actualmente por la 
Tabla 3

Beneficios tributarios y formalización del sector agrario (en miles de soles)

\begin{tabular}{lcccccc}
\hline Años & $\begin{array}{c}\text { Devolución de tributos } \\
\text { internos (DTI) }\end{array}$ & $\begin{array}{c}\text { Pagos Impuesto a } \\
\text { la Renta (PIR) })^{\prime \prime}\end{array}$ & $\begin{array}{c}\text { Gasto Tributario } \\
\text { EsSalud } \\
(\text { GTE) }\end{array}$ & $\begin{array}{c}\text { Empresas } \\
\text { Formales } \\
\text { (FT) }\end{array}$ & $\begin{array}{c}\text { Empresas } \\
\text { Exportadoras } \\
\text { (EE) }\end{array}$ & $\begin{array}{c}\mathbf{N}^{\circ} \text { Empleos } \\
\text { Formales } \\
\text { (FL) }\end{array}$ \\
\hline 2005 & 154393118 & 38032864 & 33264873 & 294 & 153 & 147,000 \\
2006 & 160879311 & 51835581 & 43123494 & 365 & 186 & 161,000 \\
2007 & 175421029 & 86362502 & 54414556 & 470 & 230 & 175,000 \\
2008 & 209416460 & 53777847 & 69886643 & 582 & 287 & 212,000 \\
2009 & 224817126 & 37657962 & 74247434 & 681 & 334 & 230,000 \\
2010 & 238999994 & 44051809 & 82642428 & 799 & 387 & 229,000 \\
2011 & 225001680 & 92216194 & 102830369 & 937 & 453 & 236,000 \\
2012 & 219387338 & 76054279 & 119962021 & 1081 & 508 & 249,000 \\
2013 & 273504326 & 96314400 & 123608384 & 1232 & 580 & 191,000 \\
2014 & 361984720 & 85474772 & 137042916 & 1419 & 652 & 211,000 \\
2015 & 408969381 & 86206398 & 152451017 & 1615 & 729 & 281,000 \\
2016 & 553702491 & 93414539 & 177473629 & 1812 & 803 & 308,000 \\
2017 & 682238283 & 91531627 & 199158565 & 2017 & 871 & 344,000 \\
2018 & 628763286 & 85443527 & 237127585 & 2233 & 941 & 413,000 \\
2019 & 826668272 & 99047677 & 253056491 & 2417 & 997 & 441,000 \\
\hline
\end{tabular}

${ }^{1 / D T I}$ representa las devoluciones en soles hechos por la SUNAT a las empresas por diferentes beneficios de devolución de impuestos como los del IGV. No se consideró el Drawback en el presente estudio por considerar como reserva tributaria las normas vigentes.

${ }^{2}$ PIR representa el monto diferencial (15\%) que deja recaudar el Estado, asumiendo una tasa promedio del 30\%. También representa los montos pagados por impuesto a la renta del sector.

${ }^{3 /}$ GTE representa el monto diferencial (5\%) que deja recaudar el Estado, asumiendo la tasa normal del $9 \%$.

${ }^{4}$ En información del rubro de empresas formales, solo se ha considerado empresas en condición de ACTIVOS por la SUNAT por acceso a la información pública.

5/La información del empleo formal ha sido promediada por año según la información de las planillas electrónicas publicadas por la SUNAT.

Fuente: Elaboración propia sobre la base de la Superintendencia Nacional de Aduanas y de Administración Tributaria (2021)

SUNAT como principales contribuyentes y figuran entre las 500 mejores exportadoras vinculadas con los grupos económicos del sector; hecho poco significativo frente a la base tributaria formal y a los más de 2 millones de productores agrarios que forman parte de la economía peruana con elevadas cifras de informalidad tributaria-laboral.

Por el lado de los beneficios laborales y la formalización laboral del sector, se evidencia que el gasto tributario (EsSalud), comparando los años durante el periodo 20052019, muestra un crecimiento del 661\%, mientras que el número de trabajadores contratados por las empresas acogidas al beneficio solo ha crecido un $200 \%$ en el mismo periodo. Ello evidencia un crecimiento desalineado, dado que a mayor gasto tributario del Estado, por consecuencia directa, debe reportarse una mayor cantidad de trabajadores con beneficios laborales declarados por las empresas formales acogidas a las normas promotoras del sector agrario, acto que no se cumple en la presente realidad, siendo relevante profundizar el análisis de dicho fenómeno empresarial-tributario.

En ese sentido, como señala Bolaños (2019), los resultados de un beneficio tributario deben analizarse desde la finalidad perseguida considerando la base total de contribuyentes de un sistema tributario, dado que representa una menor recaudación para el fisco al otorgar un determinado beneficio tributario, lo que en teoría requiere ser evaluado bajo un control estricto de efectividad y bajo el principio de proporcionalidad e idoneidad para el sistema tributario.

Considerando el espíritu de las normas tributarias promotoras de un determinado sector, el presente enfoque de análisis de los gastos tributarios debe incluir mecanismos de control implementados por los entes estatales compe- 
tentes a fin de determinar la relación entre las variables de estudio; dichos mecanismos muchas veces están ausentes en el control de los sistemas tributarios, como el peruano.

El análisis de las variables cuantitativas de la relación directa entre los dos grupos de variables canónicas detalladas en la Tabla 4, considera las cifras de los beneficios tributarios y las cifras del desarrollo económico-social.

Por otro lado, la Tabla 5 muestra la prueba de correlación canónica lineal del grupo de variables canónicas determinadas en el programa de SPSS, donde se observa que el indicador de Hotellings determina una relación directa y significativa de ambos grupos de variables ( $\mathrm{p}$-valor $<$ al 0,05). Asimismo, el indicador de la prueba de Wilks determina que los grupos de variables son diferentes y contabilizan las varianzas con un nivel de significancia estadístico resultando un p-valor < al 0,05; sin embargo, es pertinente revisar la consistencia de las relaciones en forma específica a fin de corroborar con los alcances de la teoría tributaria antes detallada.

Por su parte, la Tabla 6 muestra que el grupo 1 (PBI del sector) de la variable canónica aporta el 0,99880 de la relación, siendo relevante identificarla con la teoría tributaria y los principales efectos directos en las variables investigadas.

En tanto, la Tabla 7 evidencia que solo el primer grupo de variables está correlacionado por el valor $\mathrm{F}$ de significancia con un $\mathrm{p}$-valor $<0,05$, siendo objetivo y razonable descartar el grupo 2 y 3 por presentar un valor $\mathrm{F}$ de significancia con un p-valor $>0,05$.

La Tabla 8 evidencia que hay relaciones directas significativas entre la variable PBI del sector y las variables exportaciones del sector, formalización tributaria y pagos del impuesto a la renta empresarial, pues no existe una relación directa significativa entre los gastos EsSalud (2) y la formalización laboral (FL), al igual que entre las devoluciones de

Tabla 4

Grupos de variables canónicas planteadas

\begin{tabular}{|c|c|c|c|}
\hline \multicolumn{2}{|c|}{ Beneficios tributarios } & \multicolumn{2}{|c|}{ Desarrollo Económico- Social } \\
\hline PBIA (X1) & Producto bruto interno agrario & $\operatorname{EXP}(\mathrm{Y} 1)$ & Exportaciones del sector agrario \\
\hline GTE(X2) & Gasto tributario EsSalud & $\mathrm{FT}(\mathrm{Y} 2)$ & Formalización tributaria (régimen agrario) \\
\hline \multirow[t]{2}{*}{$\mathrm{DTI}(\mathrm{X} 3)$} & Devolución de tributos internos & $\mathrm{PIR}(\mathrm{Y} 3)$ & Pagos del impuesto a la renta empresarial \\
\hline & & $\mathrm{FL}(\mathrm{Y} 4)$ & Formalización laboral (empleos formales) \\
\hline
\end{tabular}

Fuente: Elaboración propia

Tabla 5

Correlación canónica de las variables

\begin{tabular}{cccccc}
\hline Indicador & Valor & Aprox. F & Hipotético. DF. & Error DF. & Sig. de F \\
\hline Pillais & 1,49361 & 2,47879 & 12,00 & 30,00 & 0,022 \\
Hotellings & 418,017613 & 232,32007 & 12,00 & 20,00 & 0,000 \\
Wilks & 0,00125 & 20,60081 & 12,00 & 21,46 & 0,000 \\
Roys & 0,99761 & & & & \\
\hline
\end{tabular}

Fuente: Elaboración propia sobre la base de la información procesada en el software SPSS

Tabla 6

Valores propios y correlaciones canónicas

\begin{tabular}{cccccc}
\hline Root No. & Valor propio & Pct. & Cum. Pct. & Canon Cor. & Sq. Cor \\
\hline 1 & 417,29318 & 99,78886 & 99,78886 & 0,99880 & 0,99761 \\
2 & 0,84272 & 0,20152 & 99,99038 & 0,67626 & 0,45732 \\
3 & 0,04023 & 0,00962 & 100,00000 & 0,19666 & 0,03868 \\
\hline
\end{tabular}

Fuente: Elaboración propia sobre la base de la información procesada en el software SPSS 
Tabla 7

Análisis de reducción de dimensiones

\begin{tabular}{cccccc}
\hline Roots & Wilks L. & F & Hypoth DF & Error DF & Sig. De F \\
\hline 1 To 3 & 0,00125 & 20,60081 & 12,00 & 21,46 & 0,000 \\
2 To 3 & 0,52169 & 1,15352 & 6,00 & 18,00 & 0,373 \\
3 To 3 & 0,96132 & 0,20117 & 2,00 & 10,00 & 0,821 \\
\hline
\end{tabular}

Fuente: Elaboración propia sobre la base de la información procesada en el software SPSS

Tabla 8

Correlaciones entre las variables de estudio

\begin{tabular}{cccc}
\hline Variable & $\mathbf{1}$ & $\mathbf{2}$ & $\mathbf{3}$ \\
\hline EXP & 0,99067 & 0,00588 & $-0,00903$ \\
FT & 0,99736 & $-0,05228$ & $-0,04510$ \\
PIR & 0,70761 & $-0,48919$ & 0,44469 \\
FL & 0,94372 & 0,32039 & 0,06641 \\
\hline
\end{tabular}

Nota. (1) PBI sector; (2) Gasto tributario EsSalud; (3) Devoluciones de tributos internos

Fuente: Elaboración propia sobre la base de la información procesada en el software SPSS

tributos internos y la formalización laboral, hecho que es corroborado por no cumplir con el valor F de significancia explicado en párrafos precedentes.

\section{DISCUSIÓN}

El cálculo de la correlación canónica entre los dos grupos de variables investigadas, los beneficios tributarios del sector agrario y el desarrollo económico-social peruano, evidencia una relación directa de ambos grupos de variables a nivel global en el periodo 2005-2019; sin embargo, al realizar un análisis disgregado de las correlaciones canónicas calculadas, se determina que la variable asociada resulta siendo el PBI del sector agrario, variable que se relaciona de manera directa con las exportaciones del sector, la formalización tributaria, los pagos del impuesto a la renta empresarial y la formalización laboral de trabajadores del sector. Estos resultados son concordantes con los sostenidos por Castellares et al. (2018), quienes destacan el impacto directo de las normas de promoción del sector agrario.

$\mathrm{Al}$ analizar el resto de los grupos de variables predictoras, como el gasto tributario EsSalud (GTE) y las devoluciones de tributos internos (DTI), se evidencia que no existe una relación directa significativa entre el beneficio tributario de EsSalud y la formalización laboral (FL), siendo el principal fin buscado por las normas de promoción del sector agrario. A pesar de generar empleos en la economía peruana, no son proporcionales y coherentes los resultados frente a lo que deja de recaudar el Estado peruano por una alícuota rebaja de EsSalud (4\%) para promover el empleo formal del sector, donde dichos resultados económicos son pocos significativos al compararlos con la población económicamente activa (PEA) que estuvo representada por más de 17,8 millones de personas al cierre del año 2019, cuya significancia porcentual alcanza solo a un $2,5 \%$ de aporte de empleo formal, promovido por un escaso número de empresas formales (2417) acogidas a los beneficios del sector agrario, de las cuales solo 337 agroexportadoras son consideradas principales contribuyentes por la Superintendencia Nacional de Aduanas y de Administración Tributaria (SUNAT) al cierre del año 2019 (información en tablas 2 y 3 ).

Similar evidencia muestra la correlación entre la devolución de tributos internos (DTI) y los pagos del impuesto a la renta empresarial (PIR). Al respecto, resulta cuestionable que los beneficios de devolución de impuestos (los vinculados con el IGV) concentrados en los principales contribuyentes exportadores del sector agrario sean 4,78 veces mayor a los pagos por el impuesto a la renta empresarial considerando una sumatoria de los últimos 15 años (2005 a 2019), indicador que pone en cuestionamiento los resultados positivos de las normas promotoras del sector, dado que evidencia concentración de los beneficios tributarios en pocas empresas (representa una gran ventaja financiera subsidiada para un pequeño grupo de empresas exportadoras), pues existe el agravante de que las empresas puedan pertenecer a socios vinculados o hayan cambiado de razón social de forma frecuente en los últimos años para aprovechar los beneficios o dejar de cumplir 
ciertas obligaciones tributarias-laborales, información que se ha corroborado de forma parcial por la SUNAT al seleccionar las empresas en condición de activos (información que necesita de mayor análisis causal para futuras investigaciones). Los resultados discutidos son concordantes con las conclusiones arribadas por investigaciones efectuadas en la realidad peruana (Benavente et al., 2018), en la realidad del sector agrario en Ecuador (Chuncho et al., 2021) y en Chile (Thezá et al. 2017).

Lo analizado permite concluir que si bien hay una relación positiva de los beneficios tributarios del sector agrario y el desarrollo económico-social peruano, el cálculo de las relaciones no evidencian que sea suficiente ni robusto para beneficiar al sector agrario de forma integral en su desarrollo, por lo que persiste un limitado desarrollo de la pequeña agricultura y una alta concentración en pocas empresas exportadoras. Asimismo, se muestra que existe una gran desconexión entre el tejido empresarial de las grandes y pequeñas empresas agrícolas. Los índices de informalidad tributaria y laboral son elevados (más del $80 \%$ ) al cierre del año 2019, tal como lo informa el Instituto Nacional de Estadística e Informática (2019). Esto ha sido fuente de conflictos sociales en los últimos dos años (2019-2020), promovidos por los trabajadores agrarios, quienes perciben que el régimen agrario no les otorga los beneficios justos (desarrollo económico-social) frente al crecimiento sostenido del sector agroexportador que goza de vastos beneficios tributarios que superan significativamente a su carga tributaria-laboral.

Los resultados obtenidos servirán como base para promover el rediseño de las políticas económicas-tributarias del sector agrario peruano sumido en un gran problema estructural de informalidad y limitado desarrollo económico-social (carente de gestión organizativa, tecnología y en muchos casos de infraestructura vial), constituyendo la presente investigación como fuente de conocimiento para las sucesivas reformas del sector.

Como agenda futura, al margen de los cambios introducidos por la Ley $\mathrm{N}^{\circ} 31110$ (2020), vigente desde el año 2021, que reconoce mayores derechos laborales y busca eliminar de forma progresiva los incentivos tributarios del sector agrario, es pertinente y necesario preguntarse lo siguiente: ¿Cuáles son las causas de la peligrosa concentración de los beneficios tributarios en pocas empresas agroexportadoras? y ¿qué políticas económicas-tributarias necesitan ser rediseñadas para promover un desarrollo inclusivo del fragmentado sector agrario peruano? Dichas interrogantes son necesarias para el inicio de futuras investigaciones, dado que los beneficios tributarios están financiados con los recursos tributarios del Gobierno peruano, labor que debe ser enfocada por el Ministerio de Economía y Finanzas del Perú y la Superintendencia Nacional de Aduanas y de Administración Tributaria. Es necesario generar riesgo tributario de control frente a posibles planificaciones tributarias agresivas y orientadas a aprovechar los beneficios de devolución de impuestos significativos y/o erosionar el impuesto a la renta empresarial (sobre todo por declaración de menores utilidades), dado que los recursos públicos son cada vez menos, especialmente en esta época (pandemia del COVID-19). También es pertinente considerar en el análisis cuantitativo el beneficio de devolución del drawback junto con información detallada por sector (a la cual no se ha tenido acceso en el presente estudio por considerarse como reserva tributaria por la Superintendencia Nacional de Aduanas y de Administración Tributaria), beneficio que en el año 2019 ha representado 710 millones de soles de gasto tributario que solo ha beneficiado a 1797 empresas formales.

\section{REFERENCIAS}

Badii, M., Castillo, J., Cortez, K., Wong, A., \& Villalpando, P. (2007). Análisis de correlación canónica (ACC) e investigación científica. Revista Innovaciones de Negocios, 4(8), 405-422. https://doi.org/10.29105/rinn4.8-9

Barrantes, C., Salinas, J., \& Yagüe, J. (2017). Factores que influencian el acceso a la extensión agropecuaria en Perú: Buscando modelos más inclusivos. Agricultura, Sociedad y Desarrollo, 14(2), 205-217. Recuperado de http://www.scielo.org.mx/scielo.php?script=sci abstract\&pid $=$ S1870-54722017000200205\&lng=es\&nr$\underline{\mathrm{m}=\mathrm{iso}}$

Banco Central de Reserva del Perú. (17 de agosto de 2021). Datos de Serie. Recuperado de https://estadisticas.bcrp.gob.pe/estadisticas/series/carrito/popup

Benavente, C., Camargo, P., Sarmiento, G., \& Mena, L. (2018). Evaluación del desarrollo de la agricultura periurbana y propuesta de gestión integral en el distrito de Cayma. Idesia (Arica), 36(3), 53-67. https://doi. org/10.4067/s0718-34292018005001101

Bolaños, L. (2019). El impuesto de renta en Colombia: Una mirada desde el gasto tributario. Boletín Mexicano de Derecho Comparado, 52(155), 721-756. https://doi. org/10.22201/iij.24484873e.2019.155.14946

Castellares, R., Regalado, F., \& Huarancca, M. (2018). Régimen de Promoción del sector agrario. Revista Moneda, (175), 30-35. Recuperado del sitio de Internet del Banco Central de Reserva del Perú: https://www.bcrp. gob.pe/publicaciones/revista-moneda/revista-moneda-175.html

Chuncho, L., Uriguen, P., \& Apolo, N. (2021). Ecuador: Análisis económico del desarrollo del sector agrope- 
cuario e industrial en el periodo 2000-2018. Revista Científica y Tecnológica UPSE, 8(1), 8-17. https://doi. org/10.26423/rctu.v8i1.547

Decreto Legislativo No 1053, Ley General de Aduanas. (2008). Poder Ejecutivo. Diario Oficial El Peruano, 22 de junio.

Decreto Supremo No 055-99-EF, Texto Único Ordenado de la Ley del Impuesto General a las Ventas e Impuesto Selectivo al Consumo. (1999). Poder Ejecutivo. Diario Oficial El Peruano, 5 de agosto.

Instituto Nacional de Estadística e Informática. (2019). Encuesta Nacional Agropecuaria. Recuperado de http://iinei.inei.gob.pe/microdatos/

Ley No 27360, Ley que aprueba las Normas de Promoción del Sector Agrario. (2000). Congreso de la República. Diario Oficial El Peruano, 1 de enero.

Ley $\mathrm{N}^{\circ} 31110$, Ley del Régimen Laboral Agrario y de Incentivos para el sector agrario y riego, agroexportador y agroindustrial. (2020). Congreso de la República. Diario Oficial El Peruano, 1 de enero.
Silva, C., Gómez, J., \& Nivaldo, A. (2016). Políticas de desarrollo rural en la provincia de Alicante. Mundo Agrario, 17(36), 1-22. Recuperado de http://www.mundoagrario.unlp.edu.ar/article/view/MAe034

Superintendencia Nacional de Aduanas y de Administración Tributaria. (2021). Ingresos Tributarios del Gobierno Central. Recuperado de https://www.sunat.gob. pe/estadisticasestudios/index.html

Thezá, M., Flores, D., \& Gac, D. (2017). ¿Palimpsesto de otra ruralidad? Reflexiones y propuesta. Revista Latinoamericana, 16(47), 137-164. http://dx.doi. org/10.4067/S0718-65682017000200137

Toledo, V. (2017). La política agraria del kirchnerismo. Entre el espejismo de la coexistencia y el predominio del agronegocio. Mundo Agrario, 18(37), 1-25. https:// doi.org/10.24215/15155994e 045 